\title{
FORMATION CONTROL ALGORITHM AND FORMATION SYSTEM OF MULTIPLE ROBOTS BASED ON DOUBLE MOBILE BEACONS
}

\author{
Jia-juan Fang 1 \\ ${ }^{1}$ Department of Software Engineering, Zhengzhou Technical College, Zhengzhou, Henan 450121, China \\ Email: fang_jiajuan2003@126.com
}

\begin{abstract}
Multi-robot is a new direction of robotics research and development. Aiming at formation control problem of multi - robot in unknown environment, a multi - robot algorithm based on double - moving beacon is proposed. In this paper, through the formation algorithm design and simulation verification, the realization of multi-robot control, effectively improve the accuracy of multi-robot formation. In the algorithm design, the speed of the comparison vehicle converges to $0.5 \mathrm{~m} / \mathrm{s}$ and minus $0.5 \mathrm{~m} / \mathrm{s}$, and the speed error converges to zero, with the optimal effect. This method solves the formation control problem of multi - robot in unknown environment. Simulation results show that the proposed method is feasible and effective.
\end{abstract}

Keywords: Dual-Mobile Beacon; Multi-Robot; Formation Control; Simulation Verification.

\section{Introduction}

In recent years, multiple robots have played an important role in aviation surveillance, search and rescue, underwater operations and even space exploration. Formation control of multiple mobile robots is the most basic and important problem in robot cooperation technology. The coordination and movement ability between robots is the guarantee to complete cooperative tasks such as object handling, space exploration and search and rescue.

In view of the above problems, this paper proposes a multi-robot formation algorithm and formation system based on double mobile beacons to verify the effectiveness of the algorithm through simulation verification, which provides the necessary algorithm basis for the following multirobot research.

\section{Literature Review}

Keep formation control research can be divided into the formation, formation and transformation Deng $\mathrm{Zi}$ problem, obstacle avoidance and the formation of formation, formation keeping and formation transformation can be summed up in the relative position between robot control, Agostino put forward by three to determine the relative position of relationship between robot: relative distance, relative bearing and attitude Angle [1].

This theory has become the most commonly used theoretical basis in formation control of robots. $\mathrm{Wu}$, $\mathrm{X}$., et is presented based on the decomposition strategy of multi-robot formation control method, the $\mathrm{N}$ robot in the multi-robot system is decomposed into $\mathrm{N}$ - 1 set of sub team, composed of two robots in each team, follow the robot by maintaining a expectations relative distance between the collar and a robot $\mathrm{Li}, \mathrm{d}$ and expectations relative azimuth $\Delta \psi \mathrm{i}, \mathrm{d}$ to realize own positioning[2-4].

Control the method is relatively simple, but due to the relative distance and relative azimuth measurement error, with the increase of $\mathrm{N}$, the robot will be more and more big, the relative posture errors so that the formation of failure, of multi-robot alternating crustal and others put forward the method to determine the relative position between the robot and take advantage of the robot in at least three static locator beacon, robot moving alternately, to achieve the relative position of other robots control, this method every alternate positioning error will accumulate, and the number of robots has certain requirements, alternate mobile also makes the robot speed restricted [5-8].

\section{Formation Algorithm Design and Simulation Verification}

When designing the formation controller, refer to the controller structure in the CSI control algorithm.

First, assume that the desired signal to be tracked by the system is generated by the system shown in equation (1).

$$
\begin{aligned}
& Z_{0}=A_{0} Z_{0} \\
& y_{0}=H_{0} Z_{0}
\end{aligned}
$$

Where $\mathrm{Z} 0$ is the state vector of the expected signal generation system, y0 is the expected signal to be tracked by the system, and $\mathrm{A} 0$ is the state transition 
matrix of the system, $\mathrm{HO}$ is the output matrix of the system [9].

The first part is the structure of distributed observation network shown in figure (1), as shown in equation (2).

$$
\dot{Z}_{i}=A_{0} Z_{i}+C \sum_{j \in N_{i}} a_{i j}\left(Z_{j}-Z_{i}\right)
$$

In equation (2), $\mathrm{Zi}$ is the observed state of the wheeled mobile robot numbered I. The main task of distributed observation network is to achieve the consistency of output state between multiple robots (including synchronous task and formation task).

The form of distributed observation network is not limited to the form in formula (2), but also can be used in the form of the first-order formation control algorithm and the second-order formation control algorithm in the following paragraphs [10].

In the control law designed in this paper, neighbor information is adopted $Z_{j}=\xi_{j}=\left(X_{j}, X_{j}\right)$, namely, neighbor location information and speed information. For the wheeled mobile robot numbered I, the observed state is
$Z_{j}=\xi_{j}=\left(X_{j}, \dot{X}_{j}\right)$

, that is, the expected speed and position. The function of distributed observation network is to calculate the desired position and speed information of wheeled mobile robot I according to the neighborhood position and speed information collected [11].

In the second part, the structure of the causal steady-state inverse module (CSI module) in figure 2-1 is used to calculate the expected signal of the internal dynamics of the system according to the expected position information and expected velocity information (external dynamics) of the wheeled mobile robot numbered I. Due to the non-minimum phase characteristics of the system, if the control law is only concerned with the information of position and speed, the internal dynamics of the wheeled mobile robot system will diverge. Therefore, the causal steady-state inverse module is designed to calculate the desired signal to make the internal dynamic stability of the system. The design process is described below.

The expected signal defining the whole state of the system is shown in equation (3)

$$
X_{d i}=\left(\xi_{d i}^{T}, \eta_{d i}^{T}\right)^{T}=\left(x_{d i}, \dot{x}_{d i}, \psi_{d i}, m \dot{x}_{d i}-n \dot{\psi}_{d i}\right)^{T} \in \mathfrak{R}^{4}
$$

The external dynamic of the desired value $\xi \mathrm{di}=\mathrm{Zi}$, whereas the internal dynamic of the desired value $\eta$ di is calculated using a method of constructing a differential equation to solve the deflections.

The internal dynamic state equation of the wheeled mobile robot numbered $I$ is shown in equation (4).

$$
\dot{\eta}_{\mathrm{i}}=D_{\mathrm{i}} \eta_{\mathrm{i}}+G_{\mathrm{i}} \xi_{\mathrm{i}}
$$

The desired internal vertical $\eta d i$ is the solution to the differential equation (5)

$$
\ddot{\eta}_{\mathrm{i}}+C_{\mathrm{i} 1} \eta_{\mathrm{i}}+C_{\mathrm{i} 0} \eta_{\mathrm{i}}=-\left(P_{\mathrm{i} 1} G_{\mathrm{i}} \dot{\xi}_{\mathrm{i}}+P_{\mathrm{i} 0} G_{\mathrm{i}} \xi_{\mathrm{i}}\right)
$$

Where, Ci0 and Ci1 in equation (2-5) are the values of the expected poles of the system when the state transition matrix $\mathrm{Di}$ is configured as a controllable system. While the coefficient Pi0, $\mathrm{Pi} 1 \in \mathrm{R} 4 * 4$ is calculated through equation (6).

$$
P_{\mathrm{i} 1}=\left(I_{2}+C_{i 1} D_{i}^{-1}+C_{i 0} D_{i}^{-2}\right)\left(I_{2}+P_{1} D_{i}^{-1}+P_{0} D_{i}^{-2}\right)^{-1}-I_{2}
$$

In formula (2-6), P0 and P1 are characteristic polynomial coefficients of A0 (7).

$$
P(\lambda)=\lambda^{2}+P_{1} \lambda+P_{0}
$$

Another output Udi of the causal steady-state inverse module can be obtained through equation (8).

$$
\mathrm{u}_{d i}=H_{0} A_{0} Z_{i}
$$

In the experiment, since the expected signal cannot be obtained in advance, the feed forward term udi can be set to 0 . The output of the causal steady-state inverse module is full state desired signal Xdi and feed forward term udi.

The third part is to design the state tracking controller behind the causal steady-state inverse module.

The system state equation of the wheeled mobile robot numbered I is shown in equation (9).

$$
\dot{X}_{\mathrm{i}}=A_{\mathrm{i}} X_{\mathrm{i}}+B_{\mathrm{i}} \mathrm{u}_{\mathrm{i}}
$$

The designed state tracking controller is shown in equation (10).

$$
\mathrm{u}_{\mathrm{i}}=\mathrm{u}_{d i}+k_{i}\left(X_{d i}-X_{i}\right)
$$

In equation (2-10), the control parameter Ki can be calculated by pole assignment, LQR and other methods.

\subsection{Position synchronization control algorithm}

Before the formation experiment, the synchronous movement effect of two wheeled mobile robots should be realized first. Synchronization experiment can also be regarded as a special formation. 
On the one hand, synchronization experiment is to verify the feasibility of control algorithm; on the other hand, it is to verify the correctness of data collected by the experimental platform and the connectivity of data between wheeled mobile robots.

In the experiment of position synchronization, the reference signal $\mathrm{X} 0$ of the desired position was firstly designed as the slope signal, and the structure of the controller of the desired speed V0 was shown in figure 2-1, where the designed observer was shown in equation (11).

$$
\begin{aligned}
& x_{A r}=x_{0}, v_{A r}=v_{0} \\
& \dot{x}_{B}=k_{B}\left(x_{A}-x_{B}\right)
\end{aligned}
$$

\subsection{Speed synchronization control algorithm}

Considering that the speed signal is added to the observer (12), the two wheeled mobile robots can achieve position synchronization and speed synchronization at the same time. Car A still tracks the desired signal.

$$
\begin{aligned}
& x_{A r}=x_{0}, v_{A r}=v_{0} \\
& \dot{x}_{B}=k_{B X}\left(x_{A}-x_{B}\right)+k_{B V}\left(v_{A}-v_{B}\right)
\end{aligned}
$$

\subsection{First-order formation control algorithm}

In the formation control experiment, the first order formation control law is considered to design the distributed observation network. The first order formation control law requires the body position information of the adjacent wheeled mobile robot, but does not require the body speed information of the adjacent wheeled mobile robot.

First, the communication topology of the three wheeled mobile robots is designed as shown in figure 1 , where 0 is the reference expected signal and $\mathrm{ABC}$ represents the three wheeled mobile robots respectively.

The solid line of the two-way arrow in the figure represents the interaction of position information and speed information between two wheeled mobile robots, while the dotted line of the one-way arrow represents the desired signal given by the remote control. In the experiment, the communication between two wheeled mobile robots connected by solid lines is real-time, and periodically transmits information such as their position and speed to each other. In the connection between the remote control represented by the dotted line and the wheeled mobile robot, the remote control can only be connected to one wheeled mobile robot at a certain moment, but not to three at the same time.

The signal of the remote control is unidirectional, and the expected signal transmitted is not periodic, but random. These characteristics are not considered in the simulation verification.
The communication between the wheeled mobile robot connected by solid lines is real-time, and the communication between the expected signal and the three wheeled mobile robots is also real-time.

The structure of the controller is shown in figure $2-1$, in which the observer part refers to the firstorder formation control law to design the first-order distributed observation network (13).

$$
\dot{x}_{i}=k_{i} \sum_{j \in N_{i}} a_{i j}\left(x_{j}-x_{i}-\Delta_{i j}\right)+k_{b x}\left(x_{r}-x_{i}-\Delta_{i}\right)+k_{b v}\left(\dot{x}_{r}-\dot{x}_{i}\right)
$$

Type (13), when the wheeled mobile robot I cannot receive the desired reference signal, $\mathrm{kbx}$ and $\mathrm{kbv}$ is $0, \Delta \mathrm{i}$ as a constant value, the meaning of in formation control, wheeled mobile robot body position and I expect the body keep a difference position signal, but $\Delta \mathrm{ij}=\Delta \mathrm{i}-\Delta \mathrm{j}$ meaning for wheeled mobile robot body position signal and its neighbor I wheeled mobile robot $\mathrm{j}$ keep the difference between body position signal, embodied in the experiment is to maintain constant the relative position between wheeled mobile robot.

When the communication topology is directed graph, the difference between the convergence of the system and the maintenance of formation and the undirected graph is discussed here [10].

The communication topology of directed graph is closer to that of experiment.

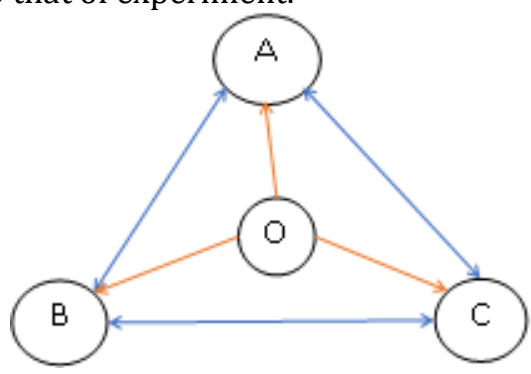

Figure 1: Simulated communication topology

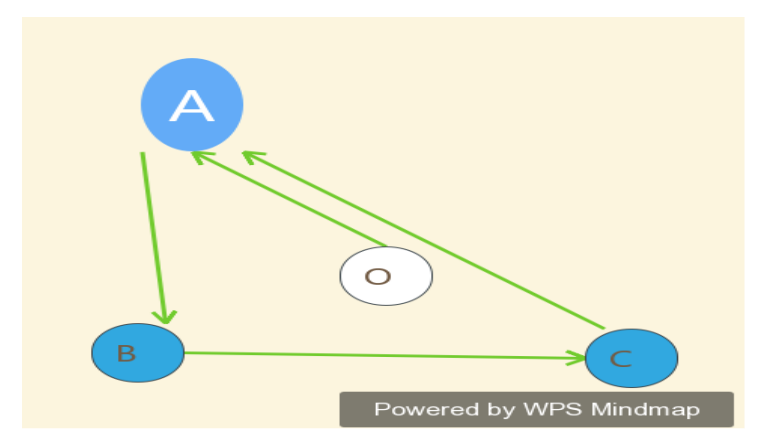

Figure 2: Communication topology of directed graph

Considered as shown in figure 2 directed graph of the communication topology, robot can receive $\mathrm{A}$ robot $C$ information and cannot receive information $B$ robots, robot $B$ will accept $A$ robot information not to receive information $C$ robots, robot can accept robot $\mathrm{B} C$ information and cannot receive $\mathrm{A}$ robot, and only $A$ robot can receive in the $A B C$ to expect signal sent by A remote control 0 . 
Compared with undirected graph, the communication topology of directed graph is closer to the actual situation in experiment.

\subsection{Second-order formation control algorithm}

As mentioned above, the first-order formation control algorithm can achieve a good formation effect, while the first-order formation control algorithm does not use the speed information of the adjacent wheeled mobile robot. The second-order formation control algorithm not only USES the position information of the adjacent wheeled mobile robot, but also USES the speed information of the adjacent wheeled mobile robot. It is worth verifying whether the experimental results will be better when using the second-order formation control algorithm [11].

The communication topology of the undirected graph is still shown in figure 12, which adopts the same communication topology as in the simulation verification of the first-order formation control algorithm.

The structure of the controller is shown in figure $2-1$, in which the observer part refers to the secondorder formation control law to design the secondorder distributed observation network (14).

$$
\ddot{x_{i}}=k_{i} \sum_{j \in N_{i}} a_{i j}\left[\left(x_{j}-x_{i}-\Delta_{i j}\right)+\gamma\left(\dot{x}_{j}-\dot{x}_{i}\right)\right]+k_{b}\left[\left(x_{r}-x_{i}-\Delta_{i}\right)+\gamma\left(x_{r}-\dot{x}_{i}\right)\right]
$$

\section{Simulation Test Results}

Among them, the first part is distributed observation network, which mainly realizes synchronous task and formation task, and the subsequent design of control algorithm is mainly aimed at this part, namely the design of distributed observer.

The second part is the causal steady-state inverse module, which aims at the non-minimum phase characteristics of the system and achieves the dynamic and stable convergence within the system.

This is also the key point to distinguish the design of the minimum phase control algorithm from the on-minimum phase control algorithm. For the minimum phase system, the internal dynamic expectation of the output of this module is the balance point of the system.

\subsection{Simulation test results of position synchronization control algorithm}

When the two cars are in position synchronization, Car B can basically track the position signal of car A, while the position error is shown in figure 2.

Finally, the position error of the two cars can converge to zero.Figure3 shows the simulation results of the speed signals of the two vehicles, and as shown in figure 4 , the speed error of the two vehicles can eventually converge to zero.

The car body inclination is shown in figure 5 .

In the process of synchronous position of two cars, the car body inclination converges.

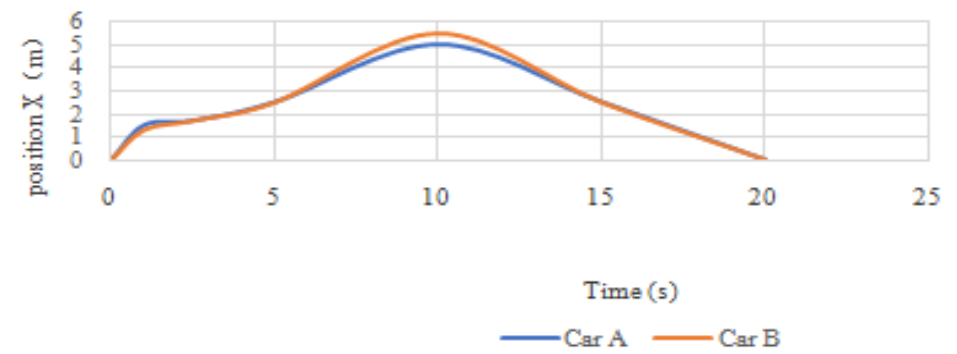

Figure 3: Simulation results of car body position when two car positions are synchronized

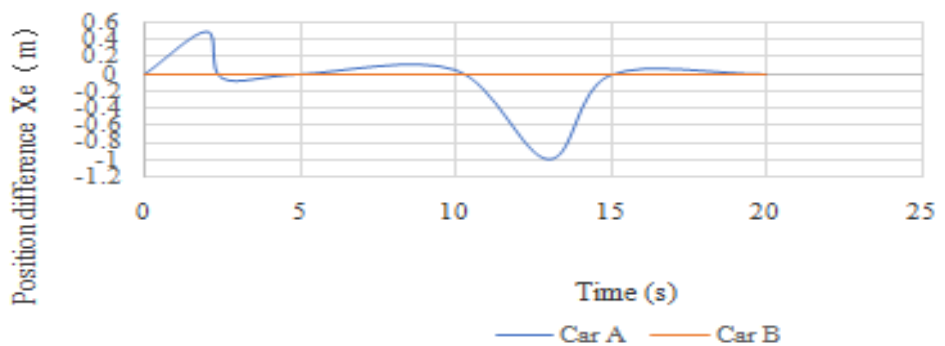

Figure 4: Simulation results of position difference between two vehicles when their positions are synchronize 


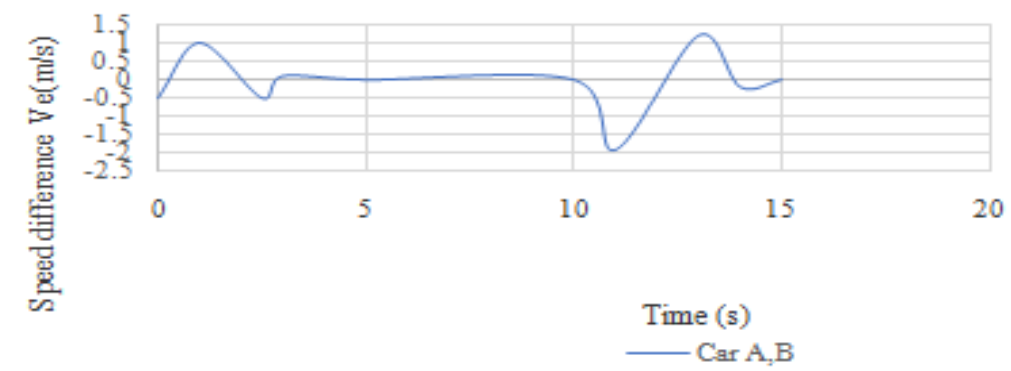

Figure 5: Simulation results of speed difference between two vehicles in position synchronization

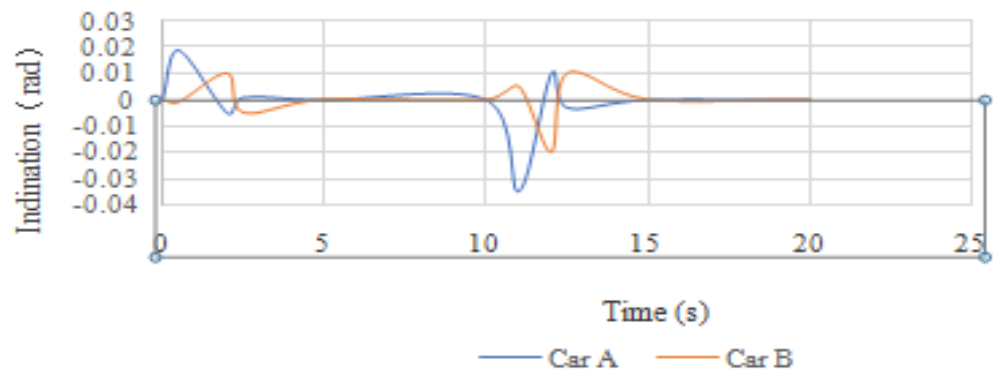

Figure 6: Simulation results of vehicle body inclination when two car positions are synchronized

\subsection{Simulation test results of speed synchronization control algorithm}

When the two cars are in speed synchronization, the simulation results of the car body speed are shown in figure 8. Car B can basically track the speed signal of car A, while the speed error is shown in figure 9.

The speed error of the two cars can eventually converge to zero. Figure 10 shows the simulation results of position signals of the two vehicles, and as shown in figure 11, the position error of the two vehicles can eventually converge to zero.

The car body inclination is shown in figure 12 . In the process of speed synchronization, the car body inclination converges.

Compared with the position synchronization of the two cars, the jitter of car B is larger and the overshoot is more serious when the speed is synchronized, and the effect of position synchronization is better.

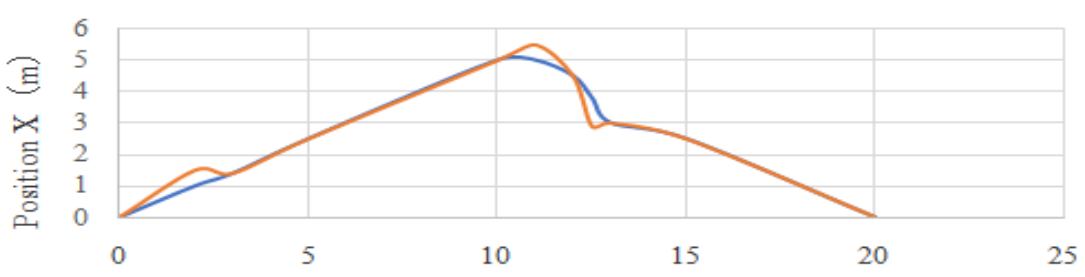

Time (s)

Car A Car B

Figure 7: Simulation results of body position of two vehicles with synchronous speed

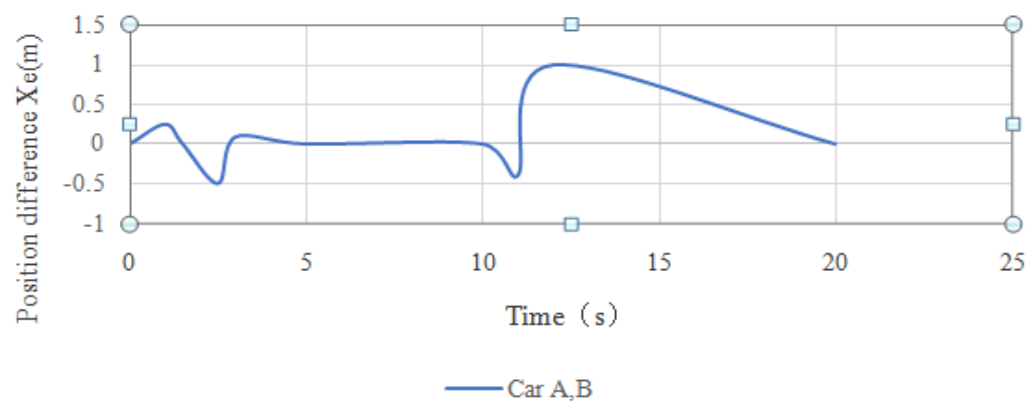

Figure 8: Simulation results of position difference between two vehicles' speed synchronization 


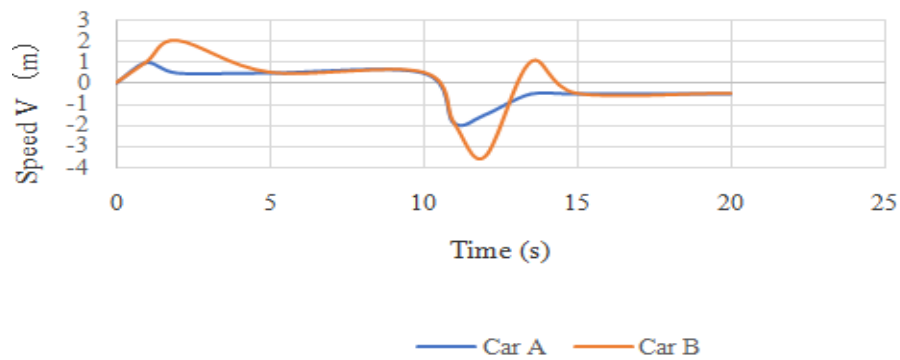

Figure 9: Simulation results of position difference between two vehicles' speed synchronization

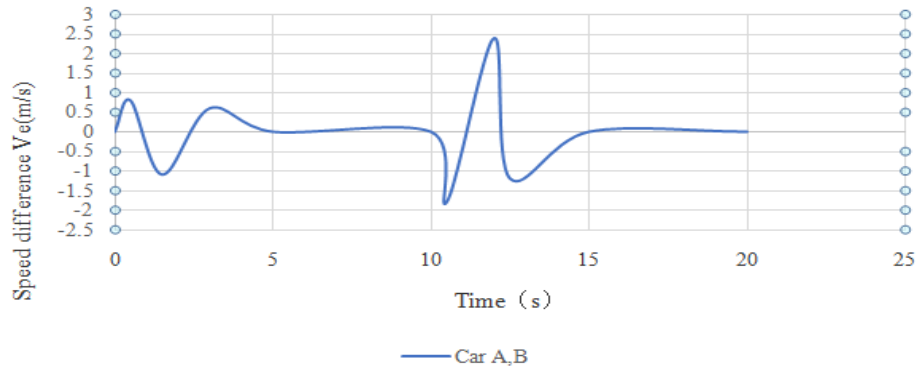

Figure 10: Simulation results of speed difference between two vehicles in speed synchronization

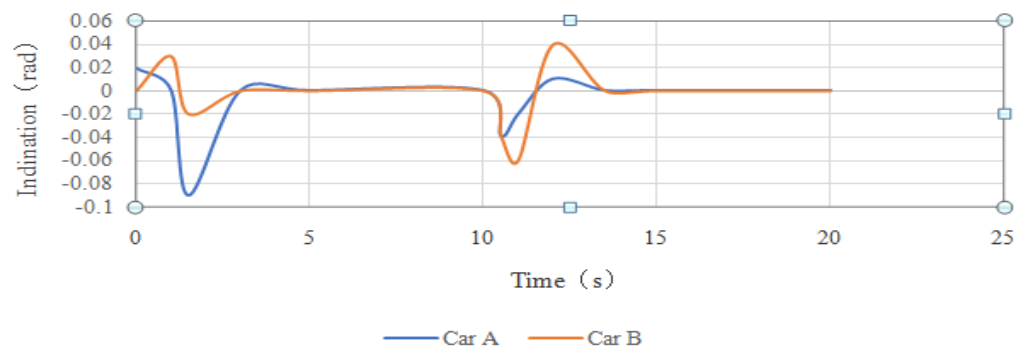

Figure 11: Simulation results of vehicle body inclination with synchronous speed between two vehicles

\subsection{Simulation test results of first-order formation control algorithm}

In the simulation, set up difference $\Delta \mathrm{ij}$ as shown in type (15).

$$
\begin{aligned}
& \Delta_{B A}=-\Delta_{A B}=4 m \\
& \Delta_{C A}=-\Delta_{A C}=2 m \\
& \Delta_{B C}=-\Delta_{C B}=2 m
\end{aligned}
$$

The simulation results of the body position of the three vehicles in the first-order formation control form $A$ formation effect that car $B$ is $2 \mathrm{~m}$ in front of car $C$ and car $C$ is $2 \mathrm{~m}$ in front of car $A$, and the position difference, $\mathrm{AB}$ position difference converges to $-4 \mathrm{~m}, \mathrm{AC}$ position difference converges to $-2 \mathrm{~m}, \mathrm{BC}$ position difference converges to $2 \mathrm{~m}$, which can also maintain the formation effect during the movement.

Simulation results of vehicle body speed $A B C$ speed successively converges to $0.5 \mathrm{~m} / \mathrm{s}$ and $0.5 \mathrm{~m} / \mathrm{s}$, and the speed error converges to zero. In the first order formation control process, the body inclination of the three vehicles converges.

In the communication topology structure of digraph, the simulation results of body position of three vehicles in first-order formation control show that the effect of formation position between three vehicles becomes worse and the desired position difference is not achieved when compared with the undigraph. Position difference.

Although the position difference between the three vehicles remains near the desired position difference, the error is large. The error between AC is the accumulation of the error between $\mathrm{AB}$ and $\mathrm{BC}$. According to the simulation results of vehicle body speed, the speed of ABC can still converge to $0.5 \mathrm{~m} / \mathrm{s}$ and $-0.5 \mathrm{~m} / \mathrm{s}$ successively, but compared with the digraph, the convergence time becomes longer and the convergence speed slows down.

The speed error, except that the convergence time becomes longer, when the speed changes, the speed of the three cars cannot remain the same, but the final error can converge to zero. Body inclination, in the case of directed graph, the consistency of the three cars becomes worse.

To sum up, the directed graph communication topology has a great influence on the first-order formation control algorithm, and the formation maintenance effect of the formation system is worse 
than that of the undirected graph communication topology.

\subsection{Simulation test results of second-order formation control algorithm}

The simulation results of the body position of the three wheeled mobile robots in the second order formation control show that the body position of the three cars in $6 \mathrm{~s}$ is A formation effect of car B at $2 \mathrm{~m}$ in front of car $\mathrm{C}$ and car $\mathrm{C}$ at $2 \mathrm{~m}$ in front of car $\mathrm{A}$.

The position difference between the three wheeled mobile robots, after $6 s$, the position difference between $\mathrm{AB}$ converges to $-4 \mathrm{~m}$, the position difference between $\mathrm{AC}$ converges to $-2 \mathrm{~m}$, and the position difference between $\mathrm{BC}$ converges to $2 \mathrm{~m}$. In the process of movement, the mutual positions between the three wheeled mobile robots can also maintain the desired formation effect.

Body speed: the body speed of $\mathrm{ABC}$ three wheeled mobile robots converges to $0.5 \mathrm{~m} / \mathrm{s}$ and $0.5 \mathrm{~m} / \mathrm{s}$ successively. Speed error: the speed error of any two of the three wheeled mobile robots converges to zero, and the speed of the three wheeled mobile robots can be synchronized during the formation movement.

In the process of the second order formation control, the body inclination of the three wheeled mobile robots converges. Compared with the firstorder formation control algorithm, the dynamic characteristics of the second-order formation control system are better, but the overshoot is larger.

In the communication topology of digraph, the simulation results of body position for the second order formation control of three vehicles show that, compared with the undigraph, the effect of formation position between three vehicles becomes worse and the desired position difference is not achieved.

Compared with the first order formation control, the influence of the second order formation control is smaller. Position difference: the position difference between the three vehicles remains close to the expected position difference, and the error is small. It can be seen that the directed graph has a smaller impact on the second-order formation control. Body speed, ABC speed can still converge to $0.5 \mathrm{~m} / \mathrm{s}$ and $0.5 \mathrm{~m} / \mathrm{s}$. The speed error, except that the convergence time becomes longer, when the speed changes, the speed of the three cars cannot remain the same, but the final error can converge to zero. Body inclination, in the case of directed graph, the consistency of the three cars becomes worse.

To sum up, the communication topology of directed graph has an impact on the second-order formation control algorithm. Compared with the communication topology of undirected graph, the formation retention effect of the formation system becomes worse. However, compared with the firstorder formation control algorithm, the communication topology of directed graph has a smaller impact on the second-order formation control algorithm.

\section{Conclusion}

Firstly, the formation controller is designed according to the non-minimum phase characteristic of wheeled mobile robot. Then, four groups of simulation verification are designed on the experimental platform of the wheeled mobile robot. The first group of experiments is to do the position synchronization simulation verification of the two wheeled mobile robot, and the second group of experiments is to do the speed synchronization simulation verification of the two wheeled mobile robot. By comparing the simulation results of position synchronization and speed synchronization, it can be found that the simulation results of position synchronization are more stable and the dynamic characteristics of speed synchronization are better.

The third group of experiments is the simulation verification of the first-order formation control algorithm of three wheeled mobile robots, and the fourth group of experiments is the simulation verification of the second-order formation control algorithm of three wheeled mobile robots.

By comparing the simulation results of the firstorder formation algorithm and the second-order formation algorithm, it can be found that the simulation results of the first-order formation control algorithm are more stable, while the dynamic characteristics of the second-order formation control algorithm are better. However, in the case of directed graph communication topology, the firstorder formation control algorithm is greatly affected, while the second-order formation control algorithm is less affected. Considering that the vehicle body speed error of wheeled mobile robot is large in the experiment, the first-order formation control algorithm of position synchronization and undirected graph is adopted in the experiment to achieve better results.

The simulation results in this paper verify the feasibility of the formation control algorithm and play a guiding role in the subsequent research.

\section{References}

[1] Li, Z., Agostino, Yao, C., Fan, K., Chu, X., \& Chen, C. L. P. (2018). Neural-dynamic optimization-based model predictive control for tracking and formation of nonholonomic multirobot systems. IEEE Transactions on Neural Networks \& Learning Systems, PP(99), 1-10.

[2] Wu, X., Wang, S., \& Xing, M. (2018). Observerbased leader-following formation control for multi-robot with obstacle avoidance. IEEE Access,PP(99), 1-1. 
[3] Qi, G., Xiang, Z., Bo, L., Hu, Z., \& Li, W. (2017). One virtual structure formation control approach based on RRT and fuzzy control algorithm. IEEE Chinese Guidance, Navigation \& Control Conference.

[4] Feng, L., Ding, Y., \& Hao, K. (2017). A neuroendocrine inspired dynamic leader selection model in formation control for multirobot system. Control \& Decision Conference.

[5] Jur, V. D. B., Patil, S., \& Alterovitz, R. (2017). Motion planning under uncertainty using iterative local optimization in belief space. Robotics Research.

[6] Schaff, C., Yunis, D., Chakrabarti, A., \& Walter, M. R. (2017). Jointly optimizing placement and inference for beacon-based localization.

[7] Ciabattoni, L., Ferracuti, F., Foresi, G., Freddi, A., Monteriù, A., \& Pagnotta, D. P. (2017). Real-time fall detection system by using mobile robots in smart homes. IEEE International Conference on Consumer Electronics-berlin.
[8] Torres-González, A., Dios, M. D., \& Ollero, A. (2017). Range-only slam for robot-sensor network cooperation. Autonomous Robots, 42(4), 1-15.

[9] Wang, H., Guo, D., Liang, X., Chen, W., Hu, G., \& Leang, K. K. (2017). Adaptive vision-based leaderfollower formation control of mobile robots. IEEE Transactions on Industrial Electronics, PP(99), 11.

[10] Roy, D., Maitra, M., \& Bhattacharya, S. (2017). Study of formation control and obstacle avoidance of swarm robots using evolutionary algorithms. IEEE International Conference on Systems.

[11] Li, Z., Wang, Y., Yao, C., Fan, K., Chu, X., \& Chen, C. L. P. (2018). Neural-dynamic optimization-based model predictive control for tracking and formation of nonholonomic multirobot systems. IEEE Transactions on Neural Networks \& Learning Systems, PP(99), 1-10. 